by significantly increased IL-4 and GM-CSF cytokine production compared to arthralgia subject $(P<0.001$ and $P=0.01)$ and $R A$-patient $(P<0.001$ and $P=0.004)$ synovial tissue. However, not all polyfunctional T-cells are equal in their pathogenic potential. Therefore, in order to identify highly pathogenic synovial T-cells, cluster analysis of flow cytometric data using the unsupervised algorithm FlowSom was performed and led to the identification of specific T-cell clusters with unique polyfunctionality characteristics. Specifically a cluster of $\mathrm{CD} 4^{+} \mathrm{CD} 8^{+}$double positive (DP) T-cells with high polyfunctionality scores was identified. Hybrid flow cytometry and imaging technique confirmed the co-expression of CD4 and CD8 by a synovial T-cell population. DP T-cells are enriched in RA-patient synovial fluid and synovial tissue and arthralgia subject synovial tissue, but are absent from HC synovial tissue. Importantly, DP T-cell synovial accumulation strongly $(\mathrm{P}=0.002)$ correlates with DAS28(CRP) of RA-patients. Initial studies utilising the novel, non-invasive FLIM technique for visualisation of cellular NAD, revealed that DP T-cells have a metabolic profile indicative of activated memory T-cells. Conclusion: These data highlight a key early loss of balance between protective and pathogenic synovial T-cell polyfunctionality and the emergence of specific, highly polyfunctional and pathogenic T-cell clusters in RA.

Disclosure of Interests: Achilleas Floudas: None declared, Nuno Neto: None declared, Mary Canavan: None declared, Trudy McGarry Employee of: Novartis, Vinod Krishna Employee of: Janssen, Sunil Nagpal Employee of: Janssen, GSK, Michael Monaghan: None declared, Douglas Veale Speakers bureau: Abbvie, Janssen, Novartis, MSD, Pfizer, UCB, Consultant of: Abbvie, Janssen, Novartis, MSD, Pfizer, UCB, Grant/research support from: Janssen, Abbvie, Pfizer, UCB, Ursula Fearon Speakers bureau: Abbvie, Grant/research support from: Janssen, Abbvie, Pfizer, UCB.

DOI: 10.1136/annrheumdis-2021-eular.2220

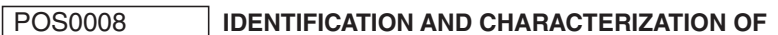 HISRS+ CD4+ T CELLS PATIENTS WITH IDIOPATHIC INFLAMMATORY MYOPATHIES}

B. Horuluoglu ${ }^{1}$, A. S. Galindo-Feria ${ }^{1}$, K. Chemin ${ }^{1}$, G. Kozhukh ${ }^{1,2}$,

A. Dubnovitsky ${ }^{1,2}$, V. Malmström ${ }^{1}$, I. E. Lundberg ${ }^{1}{ }^{1}$ Karolinska Insitutet, Division of Rheumatology, Center for Molecular Medicine, Department of Medicine, STOCKHOLM, Sweden; ${ }^{2}$ Karolinska Insitutet, Science for Life Laboratory, Department of Medicine, Stockholm, Sweden

Background: Idiopathic inflammatory myopathies (IIM) also known as myositis, are rare chronic autoimmune disorders which are represented by muscle weakness and extra-muscular features such as skin rash, interstitial lung disease (ILD) and arthritis. One of the most common autoantibodies in myositis, with a prevalence of $25-35 \%$, is the anti Jo- 1 autoantibodies, targeting the histidyl-transfer RNA synthetase (HisRS). Although the exact mechanism of how these antibodies are developed is unknown, we have previously shown that upon stimulation of both peripheral blood mononuclear cells (PBMC) and bronchoalveolar lavage fluid cells (BALF) with HisRS protein, $\mathrm{CD} 4^{+} \mathrm{T}$ cells were activated and produced inflammatory cytokines. Hitherto the presence of antigen specific autoreactive T cells has not been established in myositis, however previous studies by our group showed a strong indication of their presence with a reactivity to a specific HisRS peptide.

Objectives: The main aim of this project is to detect and characterize HisRS specific $\mathrm{CD} 4^{+} \mathrm{T}$ cells using HLA Class II tetramers. HLA Class II tetramers allow the detection of rare antigen specific CD4+ T cells and are widely used in studies of immunity, vaccine development, allergy monitoring and in autoimmunity. These cells are of specific interest to understand autoimmunity and to develop new therapies in autoimmune diseases

Methods: HLA-DRB1*03:01 monomers with selected tetanus and HisRS peptides were in-house in E.coli system. The peptides of interest were attached to the $\mathrm{N}$-terminus of the HLA b-chain via a flexible peptide linker. HLA-tetramers were assembled using a commercial fluorescently labeled streptavidin. The efficacy of the peptide-HLA tetramers was validated by stimulating PBMCs from HLA-matched healthy controls with tetanus peptide. The drequency of tetanus specific $C D 4^{+} T$ cells were detected at different time points $(6,13$ and 21 days) from the cultures using tetanus peptides bound HLA-DRB ${ }^{\star} 0301$ tetramers. The presence of tetanus specific $T$ cells was confirmed by the secretion of significantly higher IFNg levels upon re-stimulation of cells with tetanus peptide. The same protocol is applied for the HisRS-peptide tetramers. Peripheral blood cells are analysed from anti-Jo1+ and HLA-DRB1*0301 positive patients with IIM.

Results: Applying this method, our preliminary findings demonstrate the presence of HisRS ${ }^{+} \mathrm{CD}^{+}$T cells in peripheral blood from Jo- $1+$ patients $(n=3)$ using HisRS tetramers following stimulation with the respective peptide. We are now including more patient samples to confirm our findings, and further characterize their phenotype and functionalities by flow cytometry and ELISA/fluorospot assays

Conclusion: Myositis is a rare and chronic autoimmune disorder, with no currently available cure. Previous studies indicate the importance of $T$ cells in this disease. However, the phenotype, functionality and role of these cells in the disease pathogenesis has not been fully established. Characterization of this autoreactive T-cell population will help us enhance our understanding of the disease pathogenesis and thus to develop better treatment options.

Acknowledgements: This work has been supported by grants from Karolinska Instiutet Resarch Foundation, Professor Nanna Svartz Stiftelse, Hjärt-Lung Fonden and Vetenskapsrådet in Sweden.

Disclosure of Interests: Begum Horuluoglu: None declared, Angeles Shunashy Galindo-Feria: None declared, Karine Chemin: None declared, Genadiy Kozhukh: None declared, Anatoly Dubnovitsky: None declared, Vivianne Malmström: None declared, Ingrid E. Lundberg Consultant of: Consulting fees from Corbus Pharmaceuticals, Ind, Grant/research support from: Research grants from Bristol Myers Squibb and Astra Zeneca.

DOI: 10.1136/annrheumdis-2021-eular.3583

\section{POS0009 \\ THE RELATIONSHIP BETWEEN DIFFERENT IGG AND IGA ANTI-MODIFIED PROTEIN AUTOANTIBODIES IN RHEUMATOID ARTHRITIS}

C. Grönwall ${ }^{1}$, L. Liljefors ${ }^{1}$, H. Bang ${ }^{2}$, A. Hensvold ${ }^{1}$, M. Hansson ${ }^{1}$, L. Mathsson$\mathrm{Alm}^{3}$, L. Israelsson ${ }^{1}$, A. Svärd ${ }^{4}$, C. Clavel ${ }^{5}$, E. Svenungsson ${ }^{1}$, I. Gunnarsson ${ }^{1}$, G. Serre ${ }^{5}$, S. Saevarsdottir ${ }^{6}$, A. Kastbom ${ }^{4}$, L. Alfredsson ${ }^{7}$, V. Malmström ${ }^{1}$, J. Rönnelid ${ }^{8}$, A. Catrina ${ }^{1}$, K. Lundberg ${ }^{1}$, L. Klareskog ${ }^{1} .^{1}{ }^{4}$ Karolinska Institutet, Department of Medicine, Division of Rheumatology, Stockholm, Sweden; ${ }^{2}$ ORGENTEC Diagnostika GmbH, Mainz, Germany; ${ }^{3}$ Thermo Fisher Scientific, Uppsala, Sweden; ${ }^{4}$ Linköping University, Department of Biomedical and Clinical Sciences, Linköping, Sweden; ${ }^{5}$ INSERM - Université de Toulouse, Unité Différenciation Épithéliale et Autoimmunité Rhumatoïde, Toulouse, France; ${ }^{6}$ Karolinska Institutet, Department of Medicine Solna, Division of Clinical Epidemiology, Stockholm, Sweden; ${ }^{7}$ Karolinska Institutet, Institute of Environmental Medicine, Stockholm, Sweden; ${ }^{8}$ Uppsala University, Department of Immunology, Genetics and Pathology, Uppsala, Sweden

Background: Seropositive rheumatoid arthritis (RA) is characterized by the presence of rheumatoid factor (RF) and anti-citrullinated protein autoantibodies (ACPA) with different fine-specificities. Yet, other serum anti-modified protein autoantibodies (AMPA), e.g. anti-carbamylated (Carb), anti-acetylated (KAc), and anti-malondialdehyde acetaldehyde (MAA) modified protein antibodies, have been described. By using RA patient single-cell derived monoclonal antibodies we have previously shown that individual ACPA clones recognize small distinct citrulline-containing epitopes giving them extensive multireactivity when these epitopes are found in many peptides and proteins. Moreover, certain CCP2+ multireactive ACPA clones bind also to cabamylated and acetylated autoantigens [1] Objectives: To provide a comprehensive evaluation of serum $\lg G$ and $\lg A$ autoreactivity to different post-translational modifications in RA.

Methods: We analyzed 30 different IgG and IgA AMPA reactivities to modified antigens by ELISA and autoantigen arrays, in $N=1985$ newly diagnosed RA patients and population controls. The study utilized both previously established (i.e IgG and IgA CCP2; IgG ACPA fine-specificities; IgG anti-Carb fibrinogen and Carb FCS; IgG and IgA Cit/Carb/KAc/Orn(Ac)-vimentin), and novel assays (e.g. IgG anti-MAA and IgG anti-acetylated histones). Association with patient characteristics such as smoking and disease activity were explored. The newly developed assays were also evaluated in SLE disease controls and CCP2+ RA-risk individuals without arthritis.

Results: Carb and KAc reactivities by different assays were primarily seen in patients also positive for citrulline-reactivity. Modified vimentin (mod-Vim) peptides were used for direct comparison of different AMPA reactivities, revealing that IgA AMPA recognizing mod-Vim was mainly detected in subsets of patients with high IgG anti-Cit-Vim levels and a history of smoking IgG acetylation reactivity was mainly detected in a subset of patients with Cit and Carb reactivity. Anti-acetylated histone $2 B$ reactivity was RA-specific and associated with high anti-CCP2 IgG levels, multiple ACPA fine-specificities, and smoking. This reactivity was also found to be present in CCP2+ RA-risk individuals without arthritis. Our data further demonstrate that IgG autoreactivity to MAA was increased in RA compared to controls with highest levels in CCP2+RA, but was not RA-specific, and showed low correlation with other AMPA. Anti-MAA was instead associated with disease activity and was not significantly increased in CCP2+ individuals at risk of RA. Notably, RA patients could be subdivided into four different subsets based on their AMPA $\lg G$ and $\lg A$ reactivity profiles.

Conclusion: We conclude that autoantibodies exhibiting different patterns of ACPA fine-specificities as well as Carb and KAc reactivity are present in RA and may be derived from multireactive B-cell clones. Anti-Carb and anti-KAc could be considered reactivities within the "Cit-umbrella" similar to ACPA fine-specificities, while MAA is distinctly different.

\section{REFERENCES:}

[1] Sahlström P, Hansson M, Steen J, Amara K, Titcombe PJ, Forsström B, Stålesen R, Israelsson L, Piccoli L, Lundberg K, Klareskog L, Mueller DL, Catrina Al, Skriner K, Malmström V, Grönwall C. Different Hierarchies 

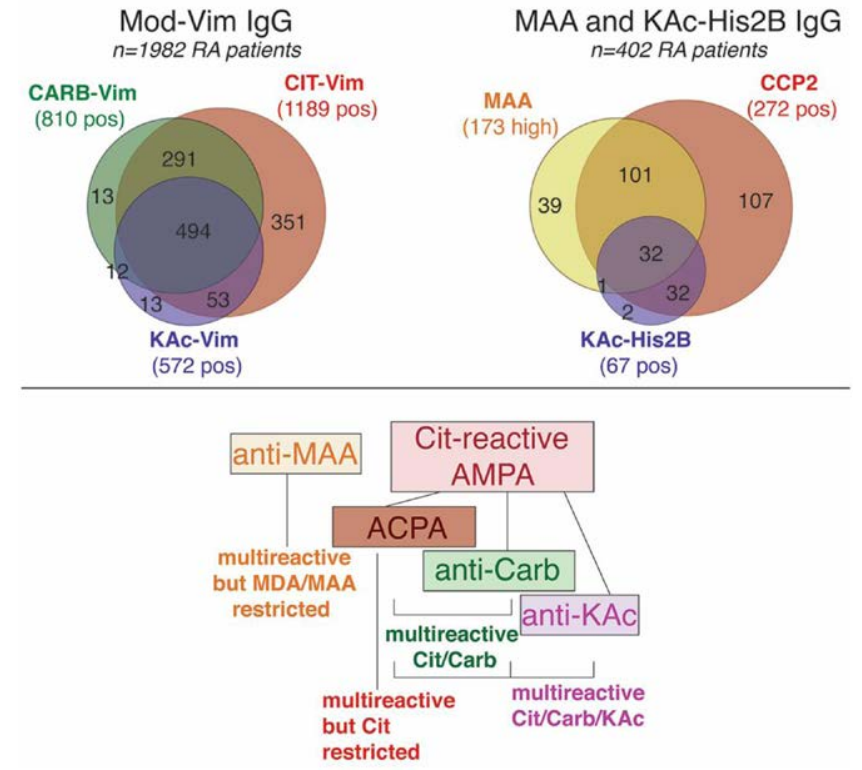

of Anti-Modified Protein Autoantibody Reactivities in Rheumatoid Arthritis. Arthritis Rheumatol. 2020 Oct;72(10):1643-1657. PMID: 32501655 Caroline Grönwall: None declared, Lisa Liljefors: None declared, Holger Bang Employee of: Employee at ORGENTEC Diagnostika $\mathrm{GmbH}$, Aase Hensvold: None declared, Monika Hansson: None declared, Linda Mathsson-Alm Employee of: Employee at Thermo Fisher Scientific, Lena Israelsson: None declared, Anna Svärd: None declared, Cyril CLAVEL: None declared, Elisabet Svenungsson: None declared, Iva Gunnarsson: None declared, Guy Serre: None declared, Saedis Saevarsdottir: None declared, Alf Kastbom: None declared, Lars Alfredsson: None declared, Vivianne Malmström: None declared, Johan Rönnelid: None declared, Anca Catrina: None declared, Karin Lundberg: None declared, Lars Klareskog: None declared

DOI: 10.1136/annrheumdis-2021-eular.3003

\section{\begin{tabular}{l|l}
\hline POS0010 & CD38+ MEMORY T CELLS ARE A FUNCTIONALLY
\end{tabular} DISTINCT SUBSET THAT IS EXPANDED IN SLE AND} ASSOCIATED WITH LUPUS NEPHRITIS

L. Ostendorf ${ }^{1,2,3}$, P. Garantziotis ${ }^{1,2}$, D. L. Wagner ${ }^{4,5,6}$, P. Durek ${ }^{7}$, F. Heinrich ${ }^{7}$, P. Enghard ${ }^{3}$, G. R. Burmester ${ }^{1}$, A. Radbruch ${ }^{8}$, M. F. Mashreghi ${ }^{7}$, F. Hiepe ${ }^{1,2}$, T. Alexander ${ }^{1,2}$. ${ }^{1}$ Charité Universitätsmedizin Berlin, Department of Rheumatology and Clinical Immunology, Berlin, Germany; ${ }^{2}$ Deutsches Rheumaforschungszentrum Berlin, Autoimmunology Laboratory, Berlin, Germany; ${ }^{3}$ Charité Universitätsmedizin Berlin, Department of Nephrology and Intensive Care Medicine, Berlin, Germany; ${ }^{4}$ Charité Universitätsmedizin Berlin, BIH Center for Regenerative Therapies (BCRT), Berlin, Germany; ${ }^{5}$ Charité Universitätsmedizin Berlin, Berlin Center for Advanced Therapies (BeCAT), Berlin, Germany; ${ }^{6}$ Charité Universitätsmedizin Berlin, Institute of Transfusion Medicine, Berlin, Germany; ${ }^{7}$ Deutsches Rheumaforschungszentrum Berlin, Therapeutic Gene Regulation Laboratory, Berlin, Germany; ${ }^{8}$ Deutsches Rheumaforschungszentrum Berlin, Cell Biology Laboratory, Berlin, Germany

Background: We recently reported the beneficial clinical responses of the anti-CD38 monoclonal antibody daratumumab in two patients with systemic lupus erythematosus (SLE) [1]. While the primary rationale for its use was the depletion of autoantibody-producing long-lived plasma cells, daratumumab may promote additional therapeutic effects on CD38-expressing T cells, but their origin, lifestyle and role in lupus pathophysiology remains elusive.

Objectives: To investigate the phenotype, transcriptional program, functional properties and clinical associations of CD4+ and CD8+CD38+ memory $T$ cells in SLE compared to healthy controls $(\mathrm{HC})$.

Methods: CD38-expression on memory $\mathrm{T}$ cell subsets was measured by flow cytometry in 65 patients with SLE and 28 healthy controls. We investigated the functional capacity of CD38+ T cells using CFSE staining and intracellular cytokine staining after polyclonal stimulation. Additionally, we performed single-cell transcriptome and T-cell-receptor sequencing of 7 SLE patients and 7 matched healthy controls, including surface protein expression analysis using CITE-seq (RNA-barcoded) antibodies.

Results: Compared to healthy controls, the frequency of CD38-expressing memory T cells in SLE was significantly increased in both CD4+ and CD8+ T cells. SLE patients with a previous or current lupus nephritis had significantly increased levels of CD8+CD38+ memory $T$ cells compared to those without history of renal involvement. CD38+ memory T cells expressed increased levels of Ki-67 and displayed higher proliferative capacity upon polyclonal stimulation than their CD38- counterparts, both in SLE patients and HC, while they showed decreased ability to secrete IFN- $\gamma$, IL-2, GM-CSF and TNF- $\alpha$. Single-cell transcriptome sequencing revealed that $\mathrm{CD} 8+\mathrm{CD} 38+$ memory $T$ cells were enriched within terminally differentiated, cytotoxic CD8 T cells, and had reduced TCR repertoire diversity compared to their CD38- counterparts. CD8+CD38+ memory $T$ cells from SLE patients had significantly higher expression of type I interferon associated genes, both compared to CD38- memory T cells from SLE patients and CD38+ cells from HCs.

Conclusion: CD38+ memory $\mathrm{T}$ cells with increased proliferative capacity but altered effector functions are significantly expanded in peripheral blood of SLE and correlate with the lupus nephritis. Although the factors mediating their generation and their precise role in the disease pathophysiology remain to be inves tigated, CD38-expressing $T$ cells may be useful as a future biomarker for lupus nephritis.

\section{REFERENCES:}

[1] Ostendorf L, Burns M, Durek P, Heinz GA, Heinrich F, Garantziotis P, et al. Targeting CD38 with Daratumumab in Refractory Systemic Lupus Erythematosus. N Engl J Med. 2020 Sep 17;383(12):1149-55.

[2] Katsuyama E, Suarez-Fueyo A, Bradley SJ, Mizui M, Marin AV, Mulki L, et al. The CD38/NAD/SIRTUIN1/EZH2 Axis Mitigates Cytotoxic CD8 T Cell Function and Identifies Patients with SLE Prone to Infections. Cell Reports. 2020 Jan;30(1):112-123.e4.

Acknowledgements: We thank our patients and healthy controls for making our research possible.

Disclosure of Interests: None declared.

DOI: 10.1136/annrheumdis-2021-eular.3104

\section{Epidemiology and treatment of pain in RMDs}

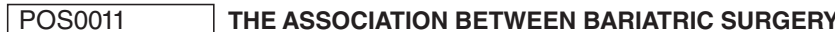 AND CARPAL TUNNEL SYNDROME: A COHORT STUDY FROM SWEDISH NATIONWIDE HEALTHCARE REGISTRIES}

J. Lane ${ }^{1}$, D. Holmberg ${ }^{2}$, A. M. Burden ${ }^{3,4}$, D. Furniss ${ }^{1}$, T. Burkard ${ }^{3} .{ }^{1}$ University of Oxford, Nuffield Department of Orthopaedics, Rheumatology, and Musculoskeletal Sciences, Oxford, United Kingdom; ${ }^{2}$ Karolinska Institutet and Karolinska University Hospital, Department of Molecular Medicine and Surgery, Stockholm, Sweden; ${ }^{3}$ ETH Zurich, Department of Chemistry and Applied Biosciences, Zurich, Switzerland; ${ }^{4}$ Maastricht University Medical Center+ Department of Clinical Pharmacy and Toxicology, Maastricht, Netherlands

Background: Carpal tunnel syndrome (CTS) is a chronic compression neuropathy caused by entrapment of the median nerve in the wrist causing pain and sensory loss. Prior observational research suggested that obesity increased the risk of CTS. However, the impact of weight loss among obese patients on CTS has not been assessed to date.

Objectives: To assess the association between bariatric surgery and CTS in a secondary care setting.

Methods: We performed a propensity score (PS)-matched cohort study using data from Swedish nationwide healthcare registries (patient registry [secondary care], causes of death registry, prescribed drug registry). Patients aged 18-79 years who underwent bariatric surgery between 2006 and 2019 were matched to up to 2 obese bariatric surgery-free patients (called unexposed patients) based on their PS. PS-matching was carried out in risk set sampling to reduce selection bias, within 4 sequential cohort entry blocks to account for time trend biases. The outcome CTS was defined as a diagnosis of CTS in secondary care or carpal tunnel decompression surgery. After a 1-year run-in period, patients were followed in an "as-treated" approach. We applied Cox proportional hazard regression to calculate hazard ratios (HR) with $95 \%$ confidence intervals (Cls) of CTS among bariatric surgery patients when compared to obese unexposed patients overall, and in subgroups of age, sex, bariatric surgery type, and by duration of follow-up.

Results: A total of 40619 bariatric surgery patients were PS-matched to 63540 obese unexposed patients. A total of $72.3 \%$ of bariatric surgery patients were women. Bariatric surgery patients had a mean age of 41.7 years and a mean follow-up of 6.8 years. All patient characteristics in obese unexposed patients were highly similar. We observed 1356 and 1938 severe CTS cases among bariatric surgery and obese unexposed patients, respectively. Bariatric surgery was not associated overall with CTS (HR of $0.98,95 \% \mathrm{Cl} 0.91-1.05)$. However the risk of CTS seemed to decrease with age at bariatric surgery - the lowest CTS risk was observed among bariatric surgery patients aged 18-34 years (HR of $0.87,95 \% \mathrm{Cl} 0.74-1.01$ ), when compared to obese unexposed patients. Sex did not modify the risk of CTS among bariatric surgery patients compared to obese 